\title{
Modeling Boundary Layer Ingestion Using a Coupled Aeropropulsive Analysis
}

\author{
Justin S. Gray* \\ NASA Glenn Research Center, Cleveland, Ohio 44139 \\ and

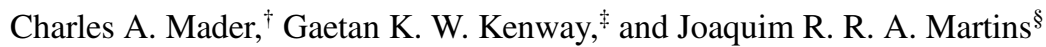 \\ University of Michigan, Ann Arbor, Michigan 48109 \\ DOI: $10.2514 / 1 . C 034601$
}

\begin{abstract}
The single-aisle turboelectric aircraft with an aft boundary layer propulsor aircraft concept is estimated to reduce fuel burn by $12 \%$ through a turboelectric propulsion system with an electrically driven boundary layer ingestion propulsor mounted on the fuselage tail cone. This motivates a more detailed study of the boundary layer ingestion propulsor design, which requires considering the fully coupled airframe propulsion integration problem. However, boundary layer ingestion studies thus far have accounted only for the aerodynamic effects on the propulsion system, or vice versa, but have not considered the two-way coupling. This work presents a new approach for building fully coupled aeropropulsive models of boundary layer ingestion propulsion systems. A onedimensional thermodynamic cycle analysis is coupled to a Reynolds-averaged Navier-Stokes simulation. This aeropropulsive model is used to assess how the propulsor design affects the overall performance for a simplified model of the single-aisle turboelectric aircraft with an aft boundary-layer propulsor. The results indicate that both propulsion and aerodynamic effects contribute equally toward the overall performance and that the fully coupled model yields substantially different results compared to the uncoupled model. Although boundary layer ingestion offers substantial fuel burn savings, it introduces propulsion-dependent aerodynamic effects that need to be accounted for.
\end{abstract}

\section{Nomenclature}

$A_{\text {ref }}=$ reference wing area

$C_{F_{\text {fuse }}}=$ force coefficient for forces generated by the fuselage

$C_{F_{\text {prop }}}=$ force coefficient for forces generated by the propulsor

$C_{F_{x}}=$ net force coefficient

$d_{\text {nac }}=$ nacelle outer diameter

$F_{\text {fuse }}=$ force integrated over the fuselage

$F_{\text {prop }}=$ force integrated over the propulsor

$F_{x}=$ net force integrated over the body

$\mathrm{FPR}=$ fan pressure ratio

$l_{\text {ref }}=$ reference length

$\dot{m}=$ mass flow rate

$p_{s}=$ static pressure

$p_{t}=$ total pressure

$\mathcal{R}=$ residual function

$T_{t} \quad=$ total temperature

$V \quad=$ air speed

$\delta=$ boundary-layer height

$\eta_{a} \quad=$ adiabatic efficiency

$\eta_{p}=$ propulsive efficiency

$\rho \quad=$ air density

\section{Subscript}

$\infty=$ freestream quantity

Superscripts

FE $=$ quantity computed at the fan-exit boundary

$\mathrm{FF}=$ quantity computed at the fan-face boundary

\section{Introduction}

A LTHOUGH boundary layer ingestion (BLI), or wake ingestion, has been well studied for marine applications since the 1960 s [1-3] , it has not yet seen widespread adoption in aircraft applications. However, recent studies have considered several new BLI-based aircraft configurations that could offer a reduction in aircraft fuel burn between 5 and $12 \%$ [4-6]. This dramatic fuel burn reduction is achieved via tight integration of the propulsion system and airframe aerodynamics, but realizing such large performance gains requires modifying the aircraft design process to account for the interactions between the two systems. The traditional approach to airframe and the propulsion system design is to design them separately and then size the engine using simple thrust scaling. This works well when the propulsion system is placed in freestream air, away from the aerodynamic influence of the airframe. In this case, it is reasonable to assume that small changes to either system do not affect the other.

This assumption is no longer valid for BLI configurations because changes to the airframe shape directly affect the flow coming into the propulsor, and conversely changes in the propulsor design directly affect the flow over the airframe. In 1947, the first theoretical study of BLI noted that BLI would introduce a significant dependence between aircraft drag and engine airflow [7]. Decades later, Smith [8] quantified this interaction from the propulsion system perspective by relating the propulsive efficiency to a set of nondimensional viscous wake parameters. He found that BLI can increase the overall propulsive efficiency above 1 , which would be considered a nonphysical result in a standalone propulsion system. These efforts highlight the main challenge of modeling aircraft performance in the presence of BLI; the aerodynamic models must be a function of propulsion mass flow, and the propulsion models must be a function of the boundary layer profile. Drela [9] proposed a control volume 
approach to the aerodynamic bookkeeping as a way to sidestep this issue. Another approach for a more unified bookkeeping approach is to use the exergy concept to quantify the overall efficiency $[10,11]$.

Despite addressing the thrust and drag accounting problem, the efforts described previously did not model the aeropropulsive coupling that is required to capture the multidisciplinary interactions that impact the overall aircraft performance. A number of studies considered the effects of BLI using techniques that capture only part of the coupling. Hardin et al. [12] found up to $10 \%$ fuel burn reduction for a BLI propulsion system with a traditional turbofan propulsion modeled using a one-dimensional (1-D) propulsion model, but their work used a fixed boundary-layer profile computed on a clean aircraft a priori with a Reynolds-averaged Navier-Stokes (RANS) solver. Kim and Liou $[13,14]$ performed a series of aerodynamic shape optimizations on a RANS model of NASA's hybrid wing-body (HWB) with BLI propulsion systems using powered boundary conditions but did not consider propulsor design variables and used a linear surrogate for the propulsion model. Blumenthal et al. [15] analyzed a tail cone thruster using an actuator disk approach to add the propulsor into a RANS simulation of the Common Research Model configurations and found that BLI offered a $14 \%$ reduction in propulsor power compared to a podded configuration. However, their work kept the diameter of and pressure change across the actuator disk constant, which amounts to a fixed propulsor design. There have also been a number of propulsion-centric studies on BLI propulsion systems, but these did not consider the changes to the flowfield due to the presence of the engine $[\underline{4}, \underline{5}, 16,17]$. All these studies are motivated by the BLI benefits arising from interdisciplinary interactions, but they make assumptions to partially decouple the analyses to make the modeling easier.

Fully coupling the propulsion and aerodynamic analyses for design optimization presents several challenges due to the cost of the analyses, the implementation of the coupling, and the large number of design variables. These challenges have prevented prior work on propulsion-airframe integration from considering the fully coupled system using models with sufficient fidelity. In this work, we tackle some of these challenges and develop the coupled analysis required for BLI design studies.

The first task toward developing a coupled analysis is to select the propulsion and aerodynamic models that capture the relevant physics. The propulsion system performance can be modeled adequately using an inexpensive 1-D thermodynamic model that represents bulk fluid properties with scalar values [18-20]. There are two main approaches to modeling viscous aerodynamics over three-dimensional (3-D) geometries. One approach is the integral boundary layer method, which couples an inviscid flow solver to an approximate boundary-layer solver [21-23]. Alternatively, we can use a fully viscous analysis, such as RANS computational fluid dynamics (CFD). For our work, we choose RANS to ensure the general applicability to complex 3-D geometries that include intersecting surfaces, highly 3 -D flows that might include vortices, and boundary layers that are not fully ingested. Although the geometry considered in the present paper is not that complex, we plan to handle more complex cases in the future.

Aerodynamic performance is highly sensitive to shape changes, and thus a parameterization with a large number of shape design variables is required [24]. RANS-based optimization with respect to large numbers of design variables has been made tractable by using gradient-based numerical optimization in conjunction with the adjoint method, which provides an efficient way to compute the required gradients [24-27].

Thus, we propose to couple a 1-D thermodynamic model of the propulsor to a RANS model of the airframe and propulsor geometry to perform BLI studies. To achieve this, we need to develop a coupling method to exchange the data between the two models and to converge the coupled solution. To leverage the efficiency of gradientbased optimization, we also plan to develop a coupled adjoint approach to compute the derivatives of the coupled system. The coupled adjoint approach, originally developed by Martins et al. $[28,29]$, has been well established for aerostructural applications [ $\underline{30}-\underline{32}]$ but has not yet been extended to aeropropulsive applications.
The ultimate goal of this research is to develop a fully coupled aeropropulsive multidisciplinary design optimization framework that can be used to study NASA's single aisle turbo electric aircraft with aft boundary layer ingestion configuration, named the STARC-ABL, and other ingegrated aeropropulsive problems. However, in this paper, we focus on the development of the coupled model and on the assessment of the coupled effects on a simplified geometry of the STARC-ABL configuration. We leverage NASA's OpenMDAO framework to perform the data exchanges and to solve the coupled system [33]. We then use the coupled model to quantify the effects of fan pressure ratio (FPR) on the overall performance of the aeropropulsive system. Our results show that, with BLI, there is a strong and mutually beneficial interaction between the airframe aerodynamics and the propulsor. Furthermore, we show that an uncoupled analysis method misses key multidisciplinary interactions, such as propulsion-dependent lift, drag, and pitching moment.

\section{STARC-ABL Aircraft Configuration}

In 2016, NASA developed a new aircraft configuration called the single-aisle turboelectric aircraft with an aft boundary layer propulsor (STARC-ABL) that incorporated BLI into an otherwise conventional configuration by adding a propulsor mounted on the tip of the tail cone. This aircraft is designed to cruise at Mach 0.72 and $37,000 \mathrm{ft}$ with a wing area of $1400 \mathrm{ft}^{2}$. A rendering of this aircraft configuration is shown in Fig. 1. STARC-ABL uses a turboelectric propulsion system with wing-mounted turbofan engines that also include electric generators to provide power to an electric motor powering the BLI propulsor.

In their initial design study, Welstead and Felder [4] conclude that this configuration could offer up to a $12 \%$ reduction in fuel burn compared to the same configuration with a conventional propulsion system. For that study, the authors assume that the BLI propulsor would always run with a fixed power input of $3500 \mathrm{hp}$, regardless of the flight speed, altitude, or throttle setting of the engine (except at very low throttle settings, where the power requirement was relaxed). Their propulsor had a FPR of 1.2 and an adiabatic efficiency of $95.6 \%$.

The total thrust of STARC-ABL underwing engines lapse more severely with altitude when compared to a conventional configuration because they must provide a fixed amount of power to the BLI propulsor. The fixed power off-take is a smaller percentage of the overall shaft power on the low-speed spool at lower altitudes. The fixed shaft power to the BLI propulsor also means that, at low altitudes, such as takeoff, the BLI propulsor does not provide as significant a portion of the overall thrust. However, as the aircraft climbs, the BLI propulsor's share of the overall thrust increases as well. According to Welstead and Felder [4], the BLI propulsor provides only $20 \%$ of the overall thrust during takeoff, but toward the end of the climb, it provides $44 \%$. This means that the performance of the propulsor is much more important at cruise than during takeoff and climb.

The large share of thrust generated by the BLI propulsor at cruise is ultimately what enables the significant mission fuel burn reduction for this configuration. Therefore, the design of this propulsor is crucial to the overall performance and viability of the STARC-ABL concept. However, as previously explained, designing a BLI

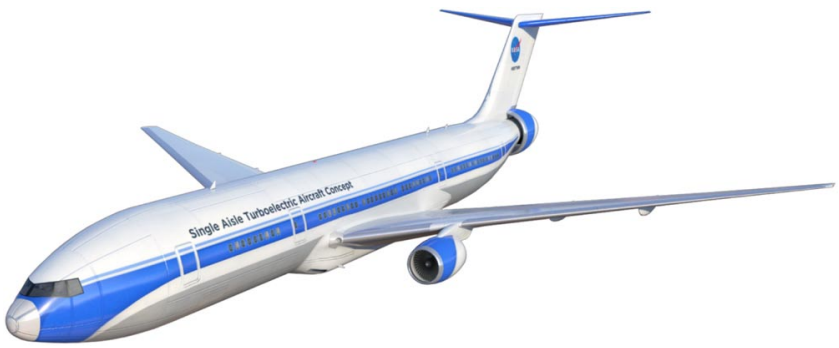

Fig. 1 NASA's STARC-ABL aircraft concept, incorporating a hybridelectric propulsion system and aft-mounted BLI propulsor [4]. 


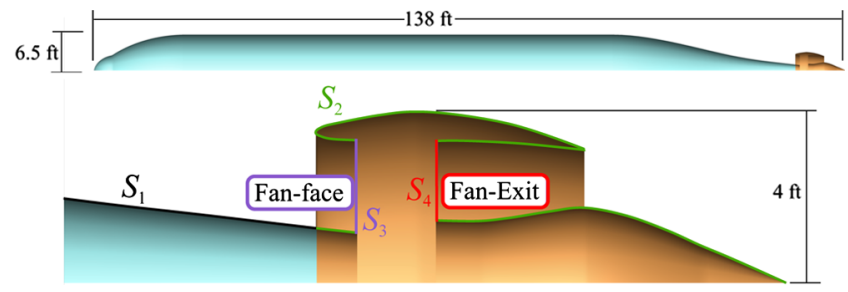

Fig. 2 Axisymmetric fuselage with aft mounted BLI propulsor representing a simplified STARC-ABL configuration.

propulsor is challenging because of the tight coupling between the propulsion and aerodynamics disciplines.

In this work, we take a step toward fully coupled BLI propulsor design optimization by performing a coupled analysis of a simplified geometry: an axisymmetric fuselage with no wings at zero angle of attack. Although this simplified geometry cannot be considered an aircraft configuration because it would not fly, it represents the simplest case where we can capture the primary interdisciplinary interactions for a BLI propulsor on the STARC-ABL configuration. A profile view of the simplified configuration, along with key dimensions, is shown in Fig. 2.

\section{Modeling}

\section{A. Propulsion Model}

The BLI propulsor is modeled using the 1-D thermodynamic cycle analysis tool pyCycle [34,35]. pyCycle, which is developed using OpenMDAO, allows for flexible modeling of propulsion systems by providing a library of different cycle elements (inlet, compressor, combustor, nozzle, and duct) that can be combined to model a specific propulsion system. pyCycle also computes adjoint analytic derivatives, which will enable design optimization with a fully coupled aeropropulsive adjoint in future work.

The propulsor model in the present work is composed of a single compressor representing the fan. The model inputs are FPR, and total temperature and pressure at the fan face. The outputs are the total temperature and pressure at the fan exit as well as the mass flow rate the for the fixed shaft power of $3500 \mathrm{hp}$. Welstead and Felder [4] selected $3500 \mathrm{hp}$ in their original work by sizing their propulsor to capture about $70 \%$ of reduced speed air. The justification for this choice was based on their analysis, which indicated that increasing the propulsor power beyond that level would achieve only marginal gains. For this work, we keep this same propulsor power, but in future work, we will allow this to vary.

The fan adiabatic efficiency $\eta_{a}$ is computed as a function of FPR. At a lower FPR, less flow turning is required, and hence a higher adiabatic efficiency can be achieved. We assume the following linear relationship between $\eta_{a}$ and FPR:

$$
\eta_{a}=1.066-0.0866 \mathrm{FPR}
$$

where the constants were chosen to give $96.2 \%$ efficiency at a $\mathrm{FPR}=1.2$ and $95 \%$ efficiency at $\mathrm{FPR}=1.4$.

\section{B. Boundary-Layer Ingestion Aerodynamic Model}

The aerodynamic model uses the RANS solver ADflow [24,25], which is an efficient viscous adjoint with an in-memory interface to Python that greatly simplifies the integration with OpenMDAO. The axisymmetric geometry of our simplified geometry allows us to use a small structured multiblock mesh with 170,000 cells to capture the relevant physics. On a desktop computer with a $3.6 \mathrm{GHz}$, four-core, Intel Core-i7 processor and $8 \mathrm{~GB}$ of memory, this mesh can be converged 10 orders of magnitude in approximately $2 \mathrm{~min}$.

The fuselage for our model was designed to be similar to that of a Boeing 737-900 in length and diameter. A free-form deformation (FFD) approach [36] was used to parameterize the BLI propulsor diameter. This was accomplished by defining an FFD that rigidly translates the top of the nacelle up or down to scale the propulsor. Figure $\underline{3}$ shows the layout of the FFD boxes that parameterize the

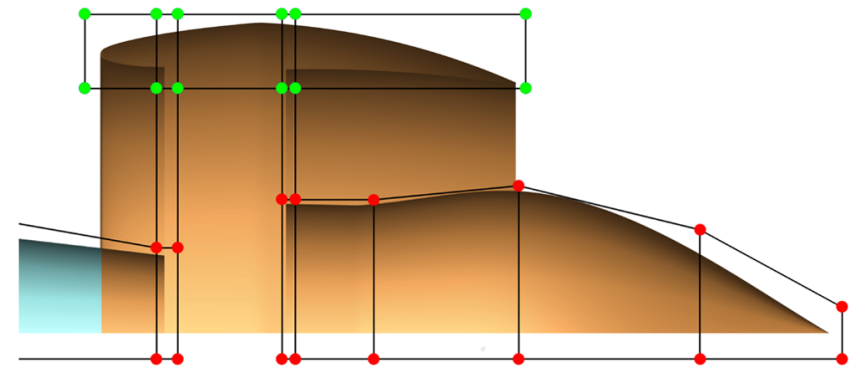

Fig. 3 Free-form deformation defining the geometry parameterization. The nodes around the outer nacelle are allowed to move, while the nodes around the fuselage and nozzle plug are fixed.

geometry, where the two upper most rows of nodes around the nacelle wall are allowed to translate up.

The propulsion-related inputs to our model are the diameter of the nacelle $\left(d_{\text {nac }}\right)$ and the two boundary conditions that account for the fan: fan-face and fan-exit boundary conditions, as shown in Fig. 2. At the fan face, we apply an outflow boundary condition (flow leaving the CFD domain), where the static pressure is specified. At the fan exit, we apply an inflow boundary condition, where the total pressure and temperature are specified

We compute the net horizontal force on the body $\left(F_{x}\right)$ by integrating the pressure and viscous forces on all solid surfaces as well as the pressure and momentum flux contributions on the fan face and fan exit, where all the contributions are resolved in the $x$ direction. This integration can be written as follows:

$$
\begin{aligned}
F_{x} & =\underbrace{\iint_{S_{1}}\left(p \hat{n}+f_{\text {visc }}\right) \cdot \hat{x} \mathrm{~d} S}_{F_{\text {fuse }}} \\
& +\underbrace{\iint_{S_{2}}\left(p \hat{n}+f_{\text {visc }}\right) \cdot \hat{x} \mathrm{~d} S-\iint_{S_{3}} \rho_{3} u_{3}^{2} \mathrm{~d} S+\iint_{S_{4}} \rho_{4} u_{4}^{2} \mathrm{~d} S}_{F_{\text {prop }}}
\end{aligned}
$$

where surfaces $S_{1}, S_{2}, S_{3}$, and $S_{4}$ are identified in Fig. 2. As indicated in this equation, we decompose the total force into $F_{\text {fuse }}$ and $F_{\text {prop }}$. We do this decomposition to facilitate the discussion of our results, but this does not imply that fuselage drag, nacelle drag, and thrust can be neatly separated. Neither of these quantities is integrated over a closed control volume, and therefore they do not represent true body forces. Furthermore, for a BLI configuration, thrust and drag are no longer independent quantities that can be separated in a meaningful way.

As part of our modeling, we also integrate the mass flow rates across both the fan face and fan exit as well as the mass-averaged total properties at the fan face. Taking a mass-average of the total properties, as opposed to an arithmetic average or area average, is important for keeping a conservative data transfer between the aerodynamic and propulsion analyses.

\section{Podded Configuration Aerodynamic Model}

To provide a consistent point of reference modeled with the same tools and assumptions as the BLI configuration, we constructed an aerodynamic model that emulates a conventional podded configuration. This model consists of two separate simulations: one for the clean fuselage, and another for the podded propulsor, as shown in Fig. 4.

For this decoupled configuration, we can assume that the aerodynamic performance of the fuselage and the propulsor are independent of each other. Using the clean fuselage, we can compute a baseline $F_{\text {fuse }}$, and then the $F_{x}$ of the podded configuration can be found by adding $F_{\text {fuse }}$ and $F_{\text {prop }}$ from a converged simulation of the independent propulsor.

Other than replacing the fuselage with a rounded spinner in the podded propulsor model, the geometry of the nacelle and boundary conditions of the BLI aerodynamic model are identical to those of the 


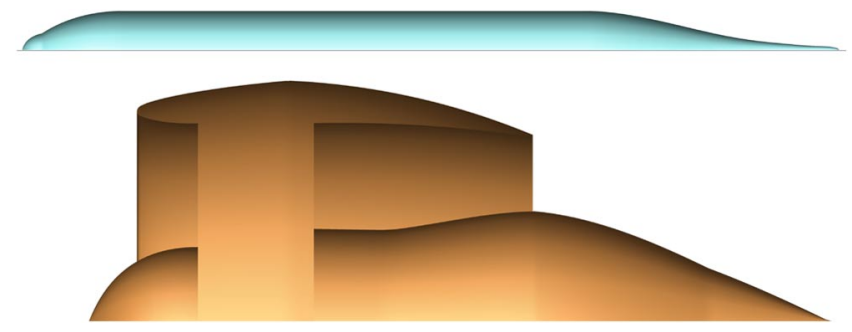

Fig. 4 Podded propulsor configuration with clean fuselage (top) and detailed view of the propulsor (bottom).

podded one. This allows the same FFD parameterization to be used for both models, and it also means that the same coupling scheme can be used for both the BLI and podded configurations.

\section{Fully Coupled Model}

Before we describe the coupling method used in this work, we should clarify what we mean by "coupled" and "uncoupled". We define a coupled model to be one where data are passed between two or more analyses in a reciprocal manner, so that some form of iteration (manual or automatic) between the analyses is performed to reach overall physical compatibility. We define uncoupled analysis to be one in which data are passed through sequentially from one model to another, requiring only one solution for each model.

There has been some recent work developing coupled aeropropulsive models for supersonic applications. Heath et al. [37] manually coupled a RANS analysis to a propulsion model by matching the flow areas and states between the two and by using the propulsion model to predict the static pressure for the fan-face boundary condition. They compared the installed performance and sonic boom for two discrete inlet designs, and the limited number of configurations made manual coupling a reasonable approach. Connolly et al. [20] developed a transient coupled model using an Euler aerodynamic model and 1-D gas-dynamics propulsion model for aeropropulsoservoelasticity applications. They iterated by passing boundary condition values at the interfaces between the two models at every time step to compute state derivatives for the time integration.

Our work uses a scheme similar to that of Connolly et al. [20], where thermodynamic states are exchanged by the two models. However, our method differs in key implementation details. Their implementation integrates the propulsion model directly into the aerodynamic solver, including the coupling terms as additional state variables to be converged with the rest of the simulation. Our method relies on the OpenMDAO framework to manage the multidisciplinary analysis and converge the simulation [33]. This approach is less intrusive because it requires no modifications to either analysis code, allowing for a more complex solver structure.

The fully coupled aeropropulsive simulation was built with a twolevel solver scheme shown in Fig. 5 [38], which was used for both podded and BLI configurations. The top level is converged by a nonlinear Gauss-Seidel solver that manages three residuals defined by the following coupling variables: fan-face total pressure $\bar{p}_{t}^{\mathrm{FF}}$, fanface total temperature $T_{t}^{\mathrm{FF}}$, and propulsor mass flow rate $\dot{m}^{\text {prop }}$. The inner level is converged using a Broyden solver that handles two additional residuals: $\mathcal{R}_{p_{s}}^{\mathrm{FF}}$ and $\mathcal{R}_{d_{\text {nac }}}$. The mass flow rate between the

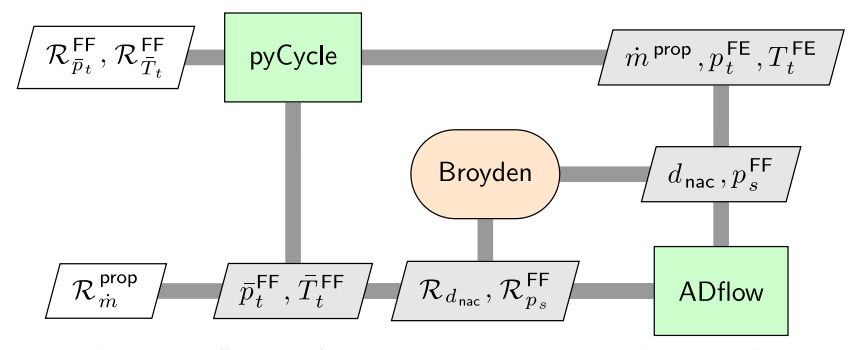

Fig. 5 XDSM [ $\underline{38}$ ] for the coupled aeropropulsive analysis. fan-face and fan-exit boundary conditions is balanced by varying fan-face static pressure $p_{s}^{\mathrm{FF}}$ to converge the static pressure residual:

$$
\mathcal{R}_{p_{s}}^{\mathrm{FF}}=\dot{m}^{\mathrm{FF}}-\dot{m}^{\mathrm{FE}}
$$

The mass flow rate between the aerodynamic and propulsion simulations is balanced by varying the nacelle diameter $d_{\text {nac }}$ to converge the nacelle diameter residual:

$$
\mathcal{R}_{d_{\text {nac }}}=\dot{m}^{\text {prop }}-\dot{m}^{\mathrm{FE}}
$$

This last residual is formulated based on the assumption of a fixed input shaft power of $3500 \mathrm{hp}$. This assumption provides a convenient way to compare different propulsor designs. Because they both have the same amount of input power, the configuration that provides the highest overall $F_{x}$ is the most efficient.

Note that an alternate reference point is also possible for which the power (and hence $\dot{m}^{\text {prop}}$ ) would be varied to achieve steady flight $\left(F_{x}=0\right)$. In this case, the design with the lowest power input to the propulsor would be the most efficient. Ultimately, this approach was discarded because part of the STARC-ABL thrust is produced by the underwing engines. It is not yet clear what the best thrust split between the underwing engines and BLI propulsor would be, and the fixed-power scheme lends itself more naturally to a problem formulation with a variable thrust split.

\section{Results}

We conducted a trade study by performing a parameter sweep of FPR from 1.2 to 1.35 and then comparing the performance of the podded configuration to that of the BLI configuration. For the podded configuration, we assume that changes to the fuselage only affect $F_{\text {fuse }}$, and changes to the propulsion system only affect $F_{\text {prop }}$. With $\mathrm{BLI}$, this assumption is no longer valid, and the performance metric is based on the net force in the axial direction $\left(F_{x}\right)$ on the combined fuselage-propulsor system. However, it is still useful to examine $F_{\text {prop }}$ and $F_{\text {fuse }}$ to understand how these quantities vary across the propulsor design space and how the net performance is achieved.

All forces are nondimensionalized as follows:

$$
C_{F}=\frac{2 F}{\rho_{\infty} V_{\infty}^{2} A_{\text {ref }}}
$$

where $A_{\text {ref }}$ is the reference wing area for the STARC-ABL aircraft. The values used in the nondimensionalization are listed in Table 1 . This table includes a reference length $l_{\text {ref }}$, whose value is the baseline outer nacelle radius and will be used to nondimensionalize the coordinates in the boundary layer.

In our convention, the sign of the force indicates the direction of action; positive values represent forward force that would cause acceleration, whereas negative values represent backward force that would cause deceleration. Note that we retain this sign convention even when breaking $F_{x}$ into $F_{\text {prop }}$ and $F_{\text {fuse }}$, and so it is expected that $F_{\text {prop }}$ be positive and $F_{\text {fuse }}$ be negative.

When working with force coefficients, it is common to refer to counts of force, which correspond to $10^{4} \times C_{F}$. The results presented later show that the BLI system outperforms the conventional podded configuration by at least twenty force counts across the range of FPR designs considered and that the aerodynamics and the propulsive improvements both contribute equally to the gains. Thus, a fully coupled analysis is necessary to accurately capture the full BLI effect.

The aerodynamic benefit of the BLI configuration is shown qualitatively in Fig. 6 , which plots contours of Mach number. The bottom image represents the clean fuselage, the middle one is a BLI configuration with $\mathrm{FPR}=1.35$, and the top one is a BLI configuration with FPR $=1.2$. As the FPR is reduced for a fixed input power to the fan, the mass flow rate increases, requiring a larger nacelle. The diameter of the nacelle has a strong influence on the height of the boundary layer on the aft fuselage. The largest nacelle, for FPR $=1.2$, also has the thickest boundary layer. The boundarylayer heights $\delta$ measured at the nacelle lip for the three configurations 
Table 1 Reference

values used in the

nondimensionalization

\begin{tabular}{lr}
\hline \hline Parameter & \multicolumn{1}{c}{ Value } \\
\hline$p_{\infty}$ & $3.834 \mathrm{psi}$ \\
$\rho_{\infty}$ & $0.0008 \mathrm{slug} / \mathrm{ft}^{3}$ \\
$V_{\infty}$ & $707.3 \mathrm{ft} / \mathrm{s}$ \\
$A_{\text {ref }}$ & $1,400 \mathrm{ft}^{2}$ \\
$l_{\text {ref }}$ & $4 \mathrm{ft}$ \\
\hline \hline
\end{tabular}

are listed in Table 2 . The boundary layer grows $15 \%$ between the podded configuration and the FPR $=1.35$ BLI configuration, whereas for the FPR $=1.2$ design, it grows by $25 \%$. This variation in the boundary-layer profile as a function of the nacelle design demonstrates a sensitivity of the aerodynamics with respect to the propulsion design variable (FPR)

\section{A. Net Force as a Function of Fan Pressure Ratio}

The calculation of $C_{F}$ must be handled slightly differently for the podded and BLI configurations. For the podded configuration, the force on the fuselage is constant and is independent of any changes to the pod. Therefore, the contribution of the fuselage can be computed at the given flight condition and then combined with the contribution from the podded propulsor for any given FPR as follows:

$$
C_{F_{x}}=C_{F_{\text {prop }}}+C_{F_{\text {fuse }}}
$$

where $C_{F_{\text {fuse }}}=-0.008321$.

For the BLI configuration, we compute the net force integration on the whole wetted surface as well as fan face and fan exit, as detailed in Eq. (2). A comparison of $C_{F_{x}}$ between the podded and BLI configurations is plotted in Fig. 7. The primary conclusion from Fig. 7 is that BLI offers an additional 24 to 27 of net force counts. That represents a $33 \%$ increase relative to the conventional podded configuration. The data also show two key trends. First, for the podded configuration, net force is insensitive to FPR, and second, the BLI configuration clearly performs better at lower FPR designs. We can gain further insight into these trends by breaking $C_{F-x}$ down into $C_{F_{\text {fuse }}}$ and $C_{F_{\text {prop }}}$ to look at the contributions from each discipline.
Table 2 Boundary-layer height at the nacelle lip for podded and BLI configurations

\begin{tabular}{lcc}
\hline \hline Configuration & $\delta, \mathrm{ft}$ & Change, $\%$ \\
\hline Podded & 4.0 & 0 \\
BLI with FPR $=1.35$ & 4.6 & 15 \\
BLI with FPR $=1.20$ & 5.0 & 25 \\
\hline \hline
\end{tabular}

\section{B. Propulsor Force as a Function of Fan Pressure Ratio}

Figure 8 shows the variation of the propulsor force with FPR for both podded and BLI configurations. For the podded configuration, where $F_{\text {fuse }}$ is constant, the variation in $C_{F-x}$ is solely due to changes in $F_{\text {prop }}$ at different FPR designs. Figure $\underline{8}$ shows that there is only a 1.4 force count variation in $F_{\text {prop }}$ as a function of FPR for the podded configuration. This result might be surprising if viewed from a purely thermodynamic perspective. Thermodynamics would predict that $F_{\text {prop }}$ would increase as the FPR is lowered due to higher propulsive efficiency and a higher adiabatic efficiency, defined by Eq. (1). However, the coupled analysis accounts for both thermodynamic and aerodynamics effects. Although the net thrust does go up, the nacelle drag also increases by nearly as much; hence, the net effect is a fairly flat response, with slightly better performance near FPR $=1.25$.

The drag increases for lower FPR designs because there is a stronger shock on the outer surface of the nacelle for the larger nacelles, as seen in Fig. 9. The presence of that shock in both configurations indicates that the nacelle geometry is not ideal. In fact, we can see that the same effect is present for the BLI configuration by looking at Fig. 6, where the area of high-speed flow is larger for the $\mathrm{FPR}=1.2$ configuration on top. Although we would normally expect the larger nacelle with a correspondingly larger fan to have more drag, it is possible that some of the adverse effects are mitigated by a better geometry. Future work will use shape optimization to allow for optimal inlet nacelle shaping for each design. However, because this suboptimal inlet design is used for all the cases presented herein, our performance comparison is still valid.

The propulsor force trend for the BLI configuration in Fig. 8 is different from the trend in Fig. 7. For FPR $=1.35$, the $\bar{B} L I$ configuration outperforms the podded configuration by 15.8 force counts. For FPR $=1.2$, the difference is 16.1 force counts. Although this variation is small, it is still opposite of the variation of $C_{F}$ with FPR. This BLI trend occurs because, as the nacelle grows, the BLI benefit decreases because a larger percentage of freestream flow is ingested, which results in increased ram drag. Hence, the BLI benefit
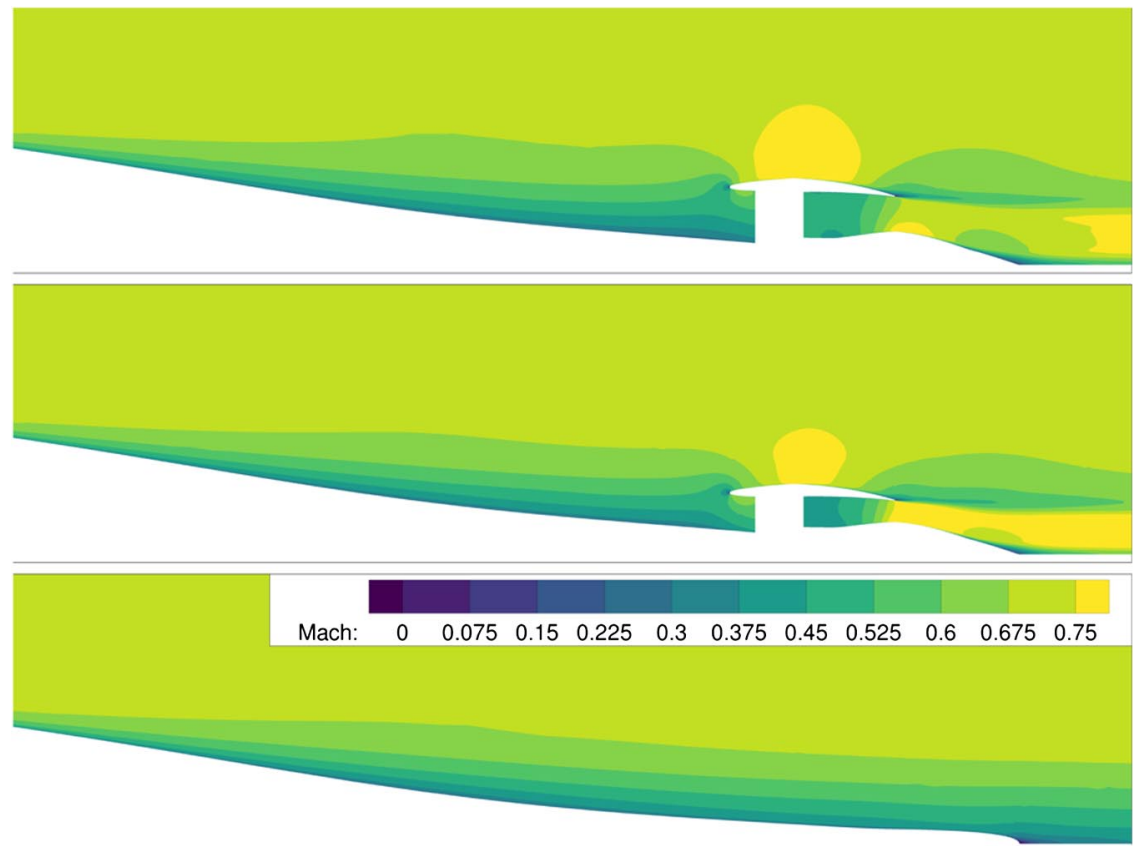

Fig. 6 Converged flow solutions for FPR $=1.2$ (top), FPR $=1.35$ (middle), and the clean fuselage (bottom) designs. 


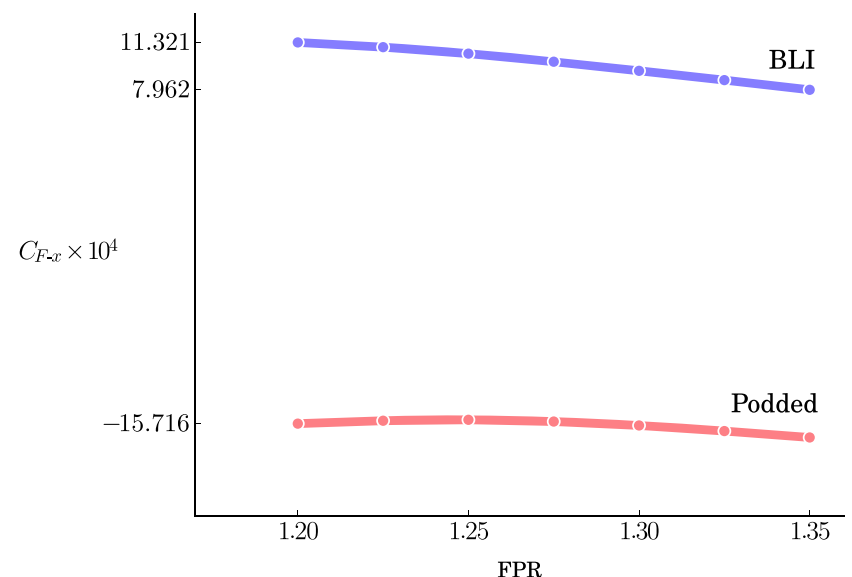

Fig. 7 Net force on the body (fuselage and propulsor) as a function of design FPR.

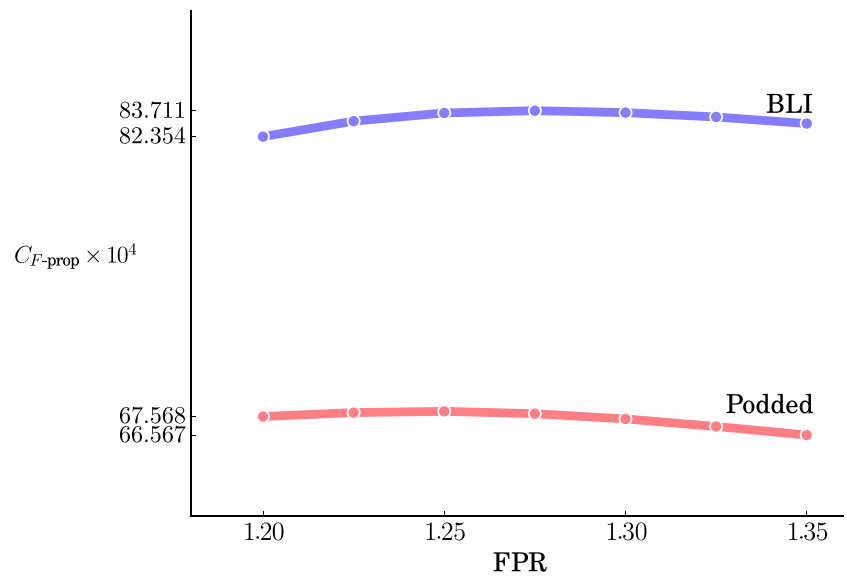

Fig. 8 Force integrated over propulsor $\left(C_{F_{\text {prop }}}\right)$ as a function of design FPR.

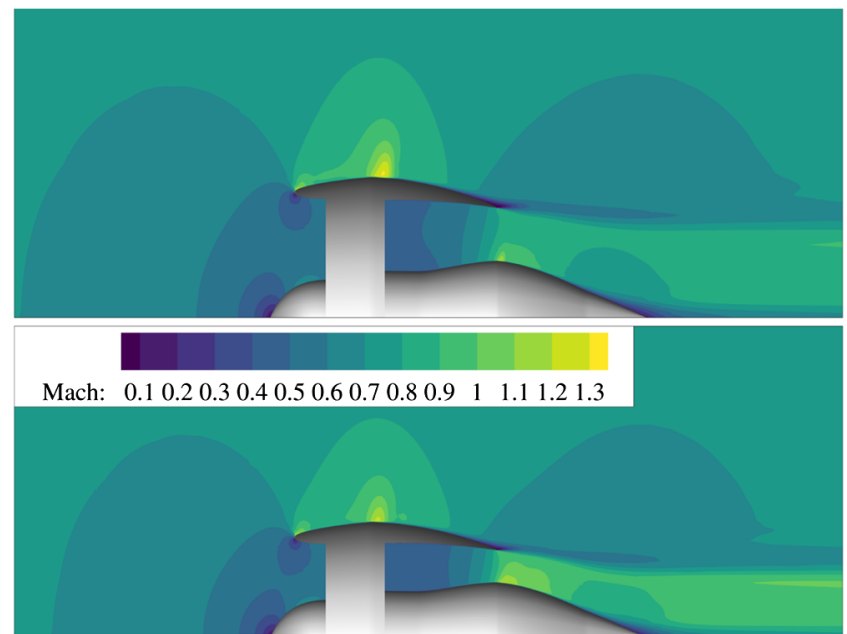

Fig. 9 Mach contours for the propulsor simulation on the FPR $=1.2$ (top) and FPR $=1.35$ (bottom) designs of the podded configuration.

to the propulsion system becomes less pronounced for lower FPR designs. Additionally, by comparing Fig. 7 with Fig. 8, we see that only considering the BLI effect on the propulsion analysis would both underpredict the benefit by between 27 and $36 \%$ and result in a different optimal FPR value.

The difference in magnitude and trend between $C_{F_{\text {nop }}}$ and $C_{F_{x}}$ highlights the importance of using a fully coupled analysis to study BLI. Another important difference between the uncoupled and

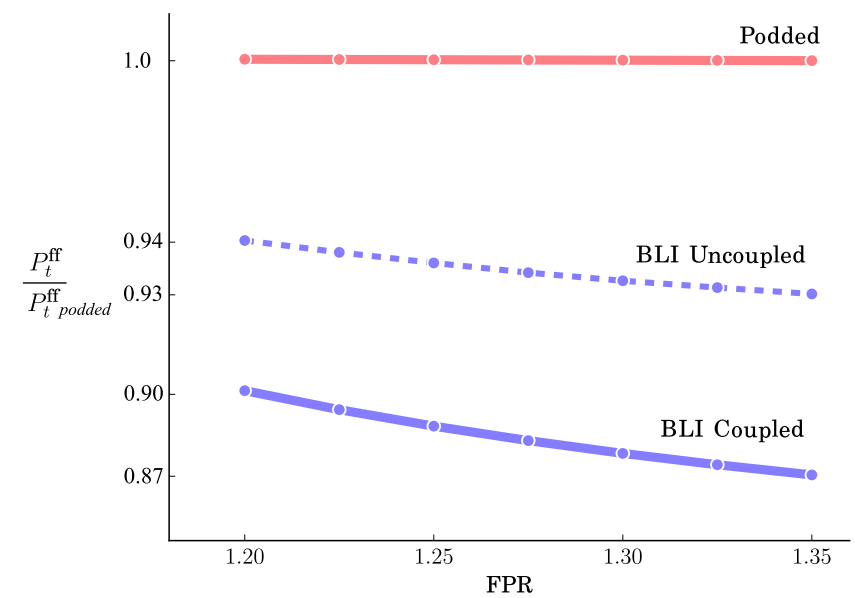

Fig. 10 Mass-averaged total pressure normalized by the value for the podded configuration as a function of FPR.

coupled modeling can be seen by examining the mass-averaged fan-face total pressure, which is defined as

$$
\bar{p}_{t}^{\mathrm{FF}}=\frac{\int_{0}^{d_{\text {nac }}} p_{t}(y) \dot{m}(y) \mathrm{d} y}{\int_{0}^{d_{\text {nac }}} \dot{m}(y) \mathrm{d} y}
$$

For an uncoupled analysis, where we assume that the aerodynamics are unchanged from the podded configuration, $p_{t}(y)$ and $\dot{m}(y)$ in Eq. (7) are extracted from the boundary-layer profile of the clean fuselage in the podded configuration and are independent of any propulsion design variable. Therefore, for uncoupled analysis, $\bar{p}_{t}^{\mathrm{FF}}$ only depends on the nacelle diameter. This type of analysis was used by Welstead and Felder [4] in their study of the STARC-ABL configuration as well as by Liu et al. [16] and Laskaridis et al. [17] in their studies of an HWB turboelectric distributed propulsion configuration.

When the analysis is fully coupled, $p_{t}^{\mathrm{FF}}$ and $\dot{m}$ are not only a function of the nacelle diameter but a function of the propulsor design as well through the FPR.

Figure 10 compares $\bar{p}_{t}$ as a function of FPR between podded configuration and the BLI configuration. The BLI results are computed using both uncoupled and coupled analyses. For the podded configuration, $\bar{p}_{t}^{\mathrm{FF}}$ is independent of FPR because the inlet just ingests from the freestream. The BLI configuration uncoupled analysis (dashed blue line) does capture the inverse relationship of $\bar{p}_{t}^{\mathrm{FF}}$ with FPR, but it overpredicts $\bar{p}_{t}^{\mathrm{FF}}$ by between 4 and $6 \%$ compared to the coupled method (solid blue line).

This overprediction is significant; for an electrically driven propulsor, a $5 \%$ lower $\bar{p}_{t}$ means that the nozzle pressure ratio decreases by the same percentage, and the result is a similar decrease in thrust. This effect would be even more pronounced for a BLI design using a conventional turbofan, where the additional total pressure loss would have a direct impact on the overall pressure ratio and thermal efficiency of the engine. Thus, it is crucial to consider the aerodynamic changes caused by the presence of the propulsor using a coupled analysis.

\section{Fuselage Force as a Function of Fan Pressure Ratio}

Figure 11 shows the aerodynamic contribution to the overall performance. The podded data are constant by definition because there is no aerodynamic interaction between the propulsor and the fuselage. The BLI configuration has better $C_{F_{\text {fuse }}}$ for all designs by at least 8.2 force counts. However, unlike the forces on the propulsor, $C_{F_{\text {fuse }}}$ for the BLI configuration exhibits a strong variation with FPR. From the FPR $=1.35$ design to the FPR $=1.2$ design, $C_{F_{\text {fuse }}}$ improves by an additional 4.0 counts. The total variation in $C_{F_{\text {fuse }}}$ is larger than the total variation in $C_{F_{\text {rop }}}$ observed in Fig. 8, indicating that the aerodynamic performance is more sensitive to FPR (a propulsor design variable) than to the propulsor performance. 


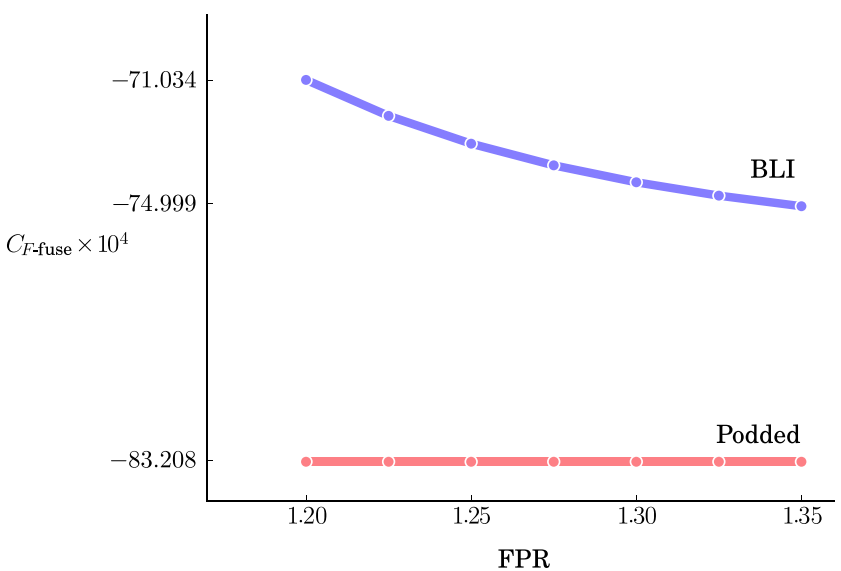

Fig. 11 Force coefficient on the fuselage $\left(C_{F_{\text {fuse }}}\right)$ as a function of design FPR.

The sensitivity of the aerodynamics to FPR in the BLI configuration is due to variations in the flowfield in the aft fuselage. We can see this effect qualitatively in Fig. 6. The actual velocity profiles for the podded configuration and the BLI configuration for FPRs of 1.35 and 1.2 are shown in Fig. 12, where all distances are normalized by the baseline nacelle outer radius $l_{\text {ref }}=4 \mathrm{ft}$. We see that the propulsor slows down the flow near the surface and that this effect extends to about one nacelle radius forward of the fan face. Also, the larger propulsor for the FPR $=1.2$ design has a more significant impact on the flow than the smaller propulsor for $\mathrm{FPR}=1.35$.

The boundary-layer profiles demonstrate that the flow on the aft fuselage is influenced by the aft propulsor and hence provide an aerodynamic justification for the results shown in Fig. 10. These profiles explain why the coupled analysis predicts a lower $p_{t}^{\mathrm{FF}}$ than the uncoupled analysis. Even for the uncoupled analysis, the dashed line in Fig. 10 shows a loss of total pressure that varies with FPR and thus with nacelle diameter as well. However, the uncoupled analysis assumes that the boundary-layer velocity profile is fixed and independent of the propulsor design. The fully coupled analysis captures the change in the boundary layer as the propulsor diameter changes, which further lowers the total pressure compared to what the uncoupled analysis predicts. The lower flow speeds in the boundary layer might also partially explain the reduced $C_{F_{\text {non }}}$ in the BLI configuration. However, the difference in boundary-layer profiles

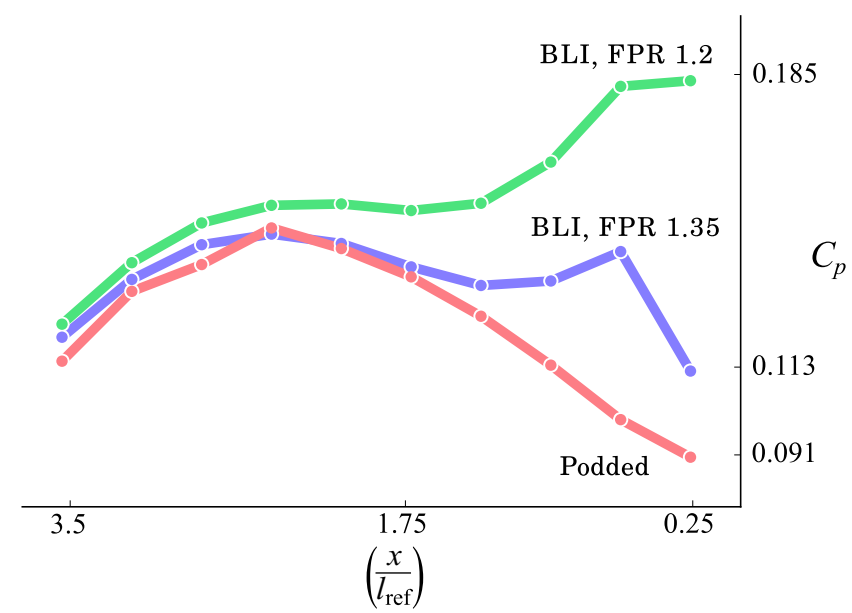

Fig. 13 Comparison of surface $C_{p}$ vs axial distance from the inlet $\left(x / l_{\text {ref }}\right)$ between the podded and BLI configurations; $l_{\text {ref }}=4 \mathrm{ft}$.

only propagates about one nacelle radius forward and qualitatively does not seem to indicate a significant reduction in viscous losses to justify the full effect seen in $C_{F_{\text {prop }}}$.

To better understand the aerodynamic cause for the change in $C_{F_{\text {prop }}}$, we examined the $C_{p}$ distribution on the surface of the aft fuselage. Figure 13 confirms that there is a significant difference in the pressure along the aft fuselage, but it also shows that the effect reaches farther upstream on the fuselage than the variation in boundary-layer profiles. $C_{p}$ near the fan face changes by $50 \%$, but even at 3.5 nacelle radii away from the inlet, there are still differences in the $C_{p}$. Hence, the surface pressure effects of the BLI propagate much farther forward than the changes in the velocity profile would suggest. Even small variations in $C_{p}$ can have a large effect on the body forces because they act over a large area. The pressure distribution shown in Fig. 13 explains the trend for the BLI configuration in Fig. 11. As the FPR decreases and the propulsor increases in diameter, the effect of the higher pressure at the fan face propagates farther upstream and further decreases $C_{F_{\text {prop }}}$.

The trends in Fig. 13 summarize the most significant finding of this work; the presence of the propulsor in a BLI configuration can have a large impact on the pressure distribution along the aerodynamic surface it is attached to. Furthermore, we can see that the details of that interaction are highly sensitive to changes in the propulsor design and shaft input power, which affects the static pressure distribution.

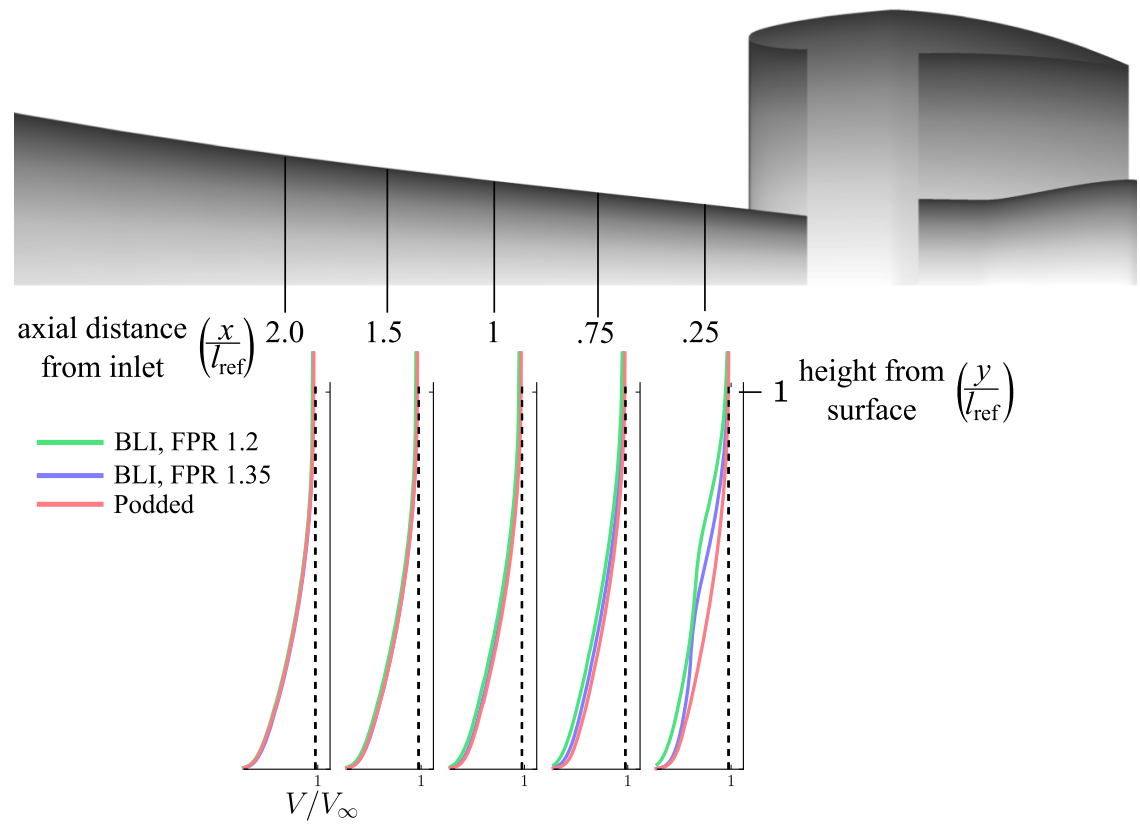

Fig. 12 Comparison of boundary layer profiles at the aft fuselage between the podded and BLI configurations; $l_{\text {ref }}=4 \mathrm{ft}$. 
Our results show that there is an inverse relationship between the change in the mean static pressure on the fuselage and FPR, but this relationship could be altered by varying other aspects of the propulsor design not considered here. The fan-face static pressure, which affects the static pressure distribution on the aft fuselage, is a function of both throttle setting and inlet design. Throttling down would increase the fan-face static pressure and thus mean static pressure, whereas throttling up would decrease it. Even for a fixed FPR and throttle setting, the inlet design could still allow an extra degree of design freedom. By making the inlet more or less diffusive, one can alter the interdisciplinary coupling between the fan-face static pressure and the aft fuselage static pressure distribution. Such inlet design changes would have an impact on propulsor performance, but the multidisciplinary effects would allow aeropropulsive trades to seek the highest overall system efficiency.

\section{Conclusions}

A new approach is presented for building coupled aeropropulsive analyses of boundary layer ingestion (BLI) propulsion systems using a 1-D cycle model and a RANS aerodynamic model. The approach was implemented in the OpenMDAO framework, using pyCycle and ADflow for the propulsion and aerodynamic analyses, respectively. A simplified version of NASA's STARC-ABL configuration was modeled, and a parameter sweep of propulsor fan pressure ratio (FPR) from 1.2 to 1.35 was performed to study the coupled performance of the aft-mounted BLI propulsor and characterize the contributions from each discipline.

The coupled analysis shows that BLI offers at least 24 force counts of increased performance relative to the podded configuration across the FPR range. This represents a $23 \%$ increase in thrust relative to the 67 force counts of thrust for the podded configuration, which clearly demonstrates the large potential for performance improvement due to BLI in this configuration.

The trends were further analyzed by breaking the net force down into aerodynamic and propulsive contributions. The breakdown of the improvements shows that 8 to 12 force counts were due to aerodynamics, and 16 counts were due to propulsion. Thus, the BLI configuration benefits from aerodynamics and propulsion are essentially of equal importance. These results demonstrate the need for a fully coupled model to predict the performance of the BLI configuration because considering only propulsion or only aerodynamics would only achieve a fraction of the possible improvement.

The BLI benefit to the propulsion system arises primarily from a decrease in incoming momentum flux (a reduction in ram drag). The thrust data presented here show that this benefit is only a weak function of FPR because of the offsetting thermodynamic improvements and nacelle drag increases. It is possible that a more-refined nacelle geometry would alleviate some of the drag penalties for the lower FPR designs shown here and thus change the trend with respect to FPR, but the general effect of decreased ram drag would persist.

The BLI benefit to the aerodynamics was due to higher mean static pressure along the aft section of the fuselage relative to the clean fuselage in the podded configuration, and this higher pressure was caused by the influence of the BLI fan. In the podded configuration, the fan-face static pressure is higher than the local static pressure on the aft section of the clean fuselage. When the propulsor is moved down onto the fuselage, the relatively higher fan-face static pressure is able to favorably alter the mean static pressure on the aft fuselage without adversely affecting the pressure forces on the propulsor.

The reduced ram drag on the propulsor combined with the increased mean static pressure along the aft fuselage combine to create the mutually beneficial effects in the BLI configuration. Although the propulsion effect was slightly larger in magnitude, the aerodynamic effect was far more sensitive to FPR. This occurred because, with a fixed input shaft power, the FPR effectively determines the nacelle diameter (i.e., a lower FPR means a larger nacelle). The diameter of the nacelle had a direct effect on how far upstream the BLI effects propagated and meant that lower FPR designs had a much larger aerodynamic effect and a strong multidisciplinary coupling.
The variation in pressure along the surface of the aft fuselage indicates that BLI propulsion systems can have a strong impact on the static pressure distribution of the associated aerodynamic surfaces. This yields a decrease in drag for the STARC-ABL configuration, but for other configurations, but for other configurations, such as the Massachusetts Institute of Technology D8 or the turboelectric distributed propulsion hybrid wing-body, one could see additional aerodynamic effects. For these aircraft, the variation in the surface pressure caused by the BLI propulsion system could cause not only propulsion-dependent drag effects but also lift and pitching moments. This means that BLI could ultimately result in throttle-dependent angle of attack and trim settings.

The overall performance of the BLI configuration depends heavily on both propulsion and aerodynamic performance, and each discipline strongly impacts the other. Both propulsion and aerodynamic performance are impacted by design choices from the other discipline. In this work, only a single propulsor design variable (FPR) was considered. However, given the strength of the coupling demonstrated by the results, the overall performance is likely to be sensitive to other variables, such as fuselage geometry, nacelle geometry, inlet design, and shaft input power. Therefore, to achieve optimal overall performance, a careful balance between aerodynamic and propulsive considerations is required. This will be addressed in future work, where this concept will be refined using design optimization with a gradient-based algorithm and coupled adjoint analytic derivatives to simultaneously optimize propulsion and aerodynamics design variables.

\section{Acknowledgments}

The authors would like to acknowledge Bret Naylor, Kenneth Moore, and the rest of the NASA Glenn OpenMDAO development team, who have spent countless hours building the framework that has enabled this research. We would also like to thank James Felder, of the NASA Glenn Research Center, for lending his time and expertise to many discussions regarding the propulsion system design of the STARC-ABL configuration. We also gratefully acknowledge NASA's Aeronautics Research Mission Directorate Transformational Tools and Technologies as well as the Advanced Air Transport Technologies projects for their continued support of this work.

\section{References}

[1] Betz, A., and Albring, W., "Introduction to the Theory of Flow Machines," Zeitschrift für Angewandte Mathematik und Mechanik, Vol. 47, No. 2, 1967, pp. 140-141.

doi:10.1002/zamm.19670470224

[2] Wislicenus, G. F., "Hydrodynamics and Propulsion of Submerged Bodies," Journal of the American Rocket Society, Vol. 30, No. 12, Dec. 1960, pp. 1140-1148. doi: $10.2514 / 8.5351$

[3] Gearhart, W. S., and Henderson, R. E., "Selection of a Propulsor for a Submersible System," Journal of Aircraft, Vol. 3, No. 1, 1966, pp. 84-90. doi:10.2514/3.59270

[4] Welstead, J. R., and Felder, J. L., "Conceptual Design of a Single-Aisle Turboelectric Commercial Transport with Fuselage Boundary Layer Ingestion," 54th AIAA Aerospace Sciences Meeting, AIAA Paper 2016$1027,2016$.

doi:10.2514/6.2016-1027

[5] Felder, J. L., Kim, H. D., and Brown, G. V., "Turboelectric Distributed Propulsion Engine Cycle Analysis for Hybrid-Wing-Body Aircraft," 47th AIAA Aerospace Sciences Meeting, AIAA Paper 2009-1132, Jan. 2009.

doi:10.2514/6.2009-1132

[6] Daggett, D. L., Kawai, R., and Friedman, D., "Blended Wing Body Systems Studies: Boundary Layer Ingestion Inlets with Active Flow Control," NASA CR-2003-212670, Dec. 2003.

[7] Smith, A. M. O., and Roberts, H. E., "The Jet Airplane Utilizing Boundary Layer Ingestion for Propulsion," Journal of Aeronautical Sciences, Vol. 14, No. 2, 1947, pp. 97-109. doi: $10.2514 / 8.1273$ 
[8] Smith, L. H., "Wake Ingestion Propulsion Benefit" Journal of Propulsion and Power, Vol. 9, No. 1, Feb. 1993, pp. 74-82. doi: $10.2514 / 3.11487$

[9] Drela, M., "Power Balance in Aerodynamic Flows," AIAA Journal, Vol. 47, No. 7, 2009, pp. 1761-1771. doi: $10.2514 / 1.42409$

[10] Arntz, A., Atinault, O., and Merlen, A., "Exergy-Based Formulation for Aircraft Aeropropulsive Performance Assessment: Theoretical Development," AIAA Journal, Vol. 53, No. 6, 2015, pp. 1627-1639. doi:10.2514/1.J053467

[11] Arntz, A., and Atinault, O., "Exergy-Based Performance Assessment of a Blended Wing-Body with Boundary-Layer Ingestion," AIAA Journal, Vol. 53, No. 12, 2015, pp. 3766-3776. doi:10.2514/1.J054072

[12] Hardin, L., Tillman, G., Sharma, O., Berton, J., and Arend, D., "Aircraft System Study of Boundary Layer Ingesting Propulsion," 48th AIAA/ ASME/SAE/ASEE Joint Propulsion Conference and Exhibit, AIAA Paper 2012-3993, July-Aug. 2012. doi: $10.2514 / 6.2012-3993$

[13] Kim, H., and Liou, M.-S., "Optimal Inlet Shape Design of N2B Hybrid Wing Body Configuration," 48th AIAAASME/SAE/ASEE Joint Propulsion Conference and Exhibit, AIAA Paper 2012-3917, July-Aug. 2012. doi:10.2514/6.2012-3917

[14] Kim, H., and Liou, M.-S., "Optimal Shape Design of Mail-Slot Nacelle on N3-X Hybrid Wing-Body Configuration," 31st AIAA Applied Aerodynamics Conference, AIAA Paper 2013-2413, 2013. doi:10.2514/6.2013-2413

[15] Blumenthal, B., Elmiligui, A. A., Geiselhart, K., Campbell, R. L., Maughmer, M. D., and Schmitz, S., "Computational Investigation of a Boundary-Layer Ingestion Propulsion System for the Common Research Model," 46th AIAA Fluid Dynamics Conference, AIAA Paper 2016-3812, 2016 doi: $10.2514 / 6.2016-3812$

[16] Liu, C., Doulgeris, G., Laskaridis, P., and Singh, R., "Thermal Cycle Analysis of Turboelectric Distributed Propulsion System with Boundary Layer Ingestion," Aerospace Science and Technology, Vol. 27, No. 1, 2013, pp. 163-170. doi:10.1016/j.ast.2012.08.003

[17] Laskaridis, P., Pachidis, V., and Pilidis, P., "Opportunities and Challenges for Distributed Propulsion and Boundary Layer Ingestion," Aircraft Engineering and Aerospace Technology, Vol. 86, No. 6, 2014, pp. $451-458$ doi:10.1108/AEAT-05-2014-0067

[18] Jones, S., "An Introduction to Thermodynamic Performance Analysis of Aircraft Gas Turbine Engine Cycles Using the Numerical Propulsion System Simulation Code," NASA TM-2007-214690, 2007.

[19] Perullo, C., Trawick, D., Clifton, W., Tai, J. C., and Mavris, D. N., "Development of a Suite of Hybrid Electric Propulsion Modeling Elements Using NPSS," Proceedings of the ASME Turbo Expo 2014: Turbine Technical Conference and Exposition, ASME Paper GT201427047, 2014

doi:10.1115/GT2014-27047

[20] Connolly, J. W., Chwalowski, P., Sanetrik, M. D., Carlson, J.-R., Silva, W. A., McNamara, J. J., and Kopasakis, G., "Towards an Aero-Propulso-Servo-Elasticity Analysis of a Commercial Supersonic Transport," 15th Dynamics Specialists Conference, AIAA Paper 20161320, 2016. doi: $10.2514 / 6.2016-1320$

[21] Drela, M., and Giles, M., "Viscous-Inviscid Analysis of Transonic and Low Reynolds Number Airfoils," AIAA Journal, Vol. 25, No. 10, 1987, pp. 1347-1355. doi: $10.2514 / 3.9789$

[22] Lock, R. C., and Williams, B. R., "Viscous-Inviscid Interactions in External Aerodynamics," Progress in Aerospace Sciences, Vol. 24, No. 2, 1987, pp. 51-171 doi:10.1016/0376-0421(87)90003-0

[23] Drela, M., "Three-Dimensional Integral Boundary Layer Formulation for General Configurations," 21st AIAA Computational Fluid Dynamics Conference, AIAA Paper 2013-2437, June 2013. doi: $10.2514 / 6.2013-2437$
[24] Lyu, Z., Kenway, G. K. W., and Martins, J. R. R. A., "Aerodynamic Shape Optimization Investigations of the Common Research Model Wing Benchmark," AIAA Journal, Vol. 53, No. 4, April 2015, pp. $968-985$. doi:10.2514/1.J053318

[25] Lyu, Z., Kenway, G. K., Paige, C., and Martins, J. R. R. A., "Automatic Differentiation Adjoint of the Reynolds-Averaged Navier-Stokes Equations with a Turbulence Model," 21st AIAA Computational Fluid Dynamics Conference, AIAA Paper 2013-2581, July 2013. doi:10.2514/6.2013-2581

[26] Lyu, Z., and Martins, J. R. R. A., "Aerodynamic Design Optimization Studies of a Blended-Wing-Body Aircraft," Journal of Aircraft, Vol. 51, No. 5, Sept. 2014, pp. 1604-1617. doi:10.2514/1.C032491

[27] Chen, S., Lyu, Z., Kenway, G. K. W., and Martins, J. R. R. A., "Aerodynamic Shape Optimization of the Common Research Model Wing-Body-Tail Configuration," Journal of Aircraft, Vol. 53, No. 1, Jan. 2016, pp. 276-293. doi: $10.2514 / 1 . \mathrm{C} 033328$

[28] Martins, J. R. R. A., Alonso, J. J., and Reuther, J. J., "High-Fidelity Aerostructural Design Optimization of a Supersonic Business Jet," Journal of Aircraft, Vol. 41, No. 3, 2004, pp. 523-530. doi:10.2514/1.11478

[29] Martins, J. R. R. A., Alonso, J. J., and Reuther, J. J., "A Coupled-Adjoint Sensitivity Analysis Method for High-Fidelity Aero-Structural Design," Optimization and Engineering, Vol. 6, No. 1, March 2005, pp. 33-62. doi:10.1023/B:OPTE.0000048536.47956.62

[30] Kenway, G. K. W., Kennedy, G. J., and Martins, J. R. R. A., "Scalable Parallel Approach for High-Fidelity Steady-State Aeroelastic Analysis and Adjoint Derivative Computations," AIAA Journal, Vol. 52, No. 5, 2014, pp. 935-951. doi:10.2514/1.J052255

[31] Kenway, G. K. W., and Martins, J. R. R. A., "Multipoint High-Fidelity Aerostructural Optimization of a Transport Aircraft Configuration," Journal of Aircraft, Vol. 51, No. 1, 2014, pp. 144-160. doi:10.2514/1.C032150

[32] Liem, R. P., Kenway, G. K. W., and Martins, J. R. R. A., "Multimission Aircraft Fuel Burn Minimization via Multipoint Aerostructural Optimization," AIAA Journal, Vol. 53, No. 1, 2015, pp. 104-122. doi:10.2514/1.J052940

[33] Gray, J. S., Hearn, T. A., Moore, K. T., Hwang, J. T., Martins, J. R. R. A., and Ning, A., "Automatic Evaluation of Multidisciplinary Derivatives Using a Graph-Based Problem Formulation in OpenMDAO," 15th AIAA/ISSMO Multidisciplinary Analysis and Optimization Conference, AIAA Paper 2014-2042, Aug. 2014. doi:10.2514/6.2014-2042

[34] Hearn, D. T., Hendricks, E., Chin, J., Gray, J., and Moore, D. K. T., "Optimization of Turbine Engine Cycle Analysis with Analytic Derivatives," 17th AIAA/ISSMO Multidisciplinary Analysis and Optimization Conference, AIAA Paper 2016-4297, 2016. doi: $10.2514 / 6.2016-4297$

[35] Gray, J., Chin, J., Hearn, T., Hendricks, E., Lavelle, T., and Martins, J. R. R. A., "Thermodynamics for Gas Turbine Cycles with Analytic Derivatives in OpenMDAO," 57th AIAA/ASCE/AHS/ASC Structures, Structural Dynamics, and Materials Conference, AIAA Paper 2016$0669,2016$. doi:10.2514/6.2016-0669

[36] Kenway, G. K., Kennedy, G. J., and Martins, J. R. R. A., "A CAD-Free Approach to High-Fidelity Aerostructural Optimization," 13th AIAA/ ISSMO Multidisciplinary Analysis Optimization Conference, AIAA Paper 2010-9231, Sept. 2010. doi:10.2514/6.2010-9231

[37] Heath, C. M., Slater, J. W., and Rallabhandi, S. K., "Inlet Trade Study for a Low-Boom Aircraft Demonstrator," Journal of Aircraft, Vol. 54, No. 4, 2017, pp. 1283-1293. doi:10.2514/1.C034036

[38] Lambe, A. B., and Martins, J. R. R. A., "Extensions to the Design Structure Matrix for the Description of Multidisciplinary Design, Analysis, and Optimization Processes," Structural and Multidisciplinary Optimization, Vol. 46, No. 2, 2012, pp. 273-284. doi:10.1007/s00158-012-0763-y 